\title{
Near infrared fluorescent chlorophyll nanoscale liposomes for sentinel lymph node mapping
}

\author{
This article was published in the following Dove Press journal: \\ International Journal of Nanomedicine \\ 19 June 2012 \\ Number of times this article has been viewed
}

\author{
Lina $\operatorname{Fan}^{1, *}$ \\ Qiang Wu ${ }^{1, *}$ \\ Maoquan Chu' ${ }^{1,2}$ \\ 'School of Life Science and Technology, \\ ${ }^{2}$ The Institute for Advanced Materials \\ and Nano Biomedicine Tongji \\ University, Shanghai, People's Republic \\ of China \\ *These authors contributed equally \\ to this work
}

Correspondence: Maoquan Chu The Institute for Advanced Materials and Nano Biomedicine, Tongji University, Shanghai 200092, People's Republic of China

Tel +862165982586

Fax +86 2I 65988653

Email mqchu98@tongji.edu.cn
Background: Sentinel lymph node (SLN) mapping using in vivo near infrared fluorescence imaging has attracted great attention during the past few years. Here we report on the early use of poorly water-soluble chlorophyll with near infrared fluorescence extracted from the leaf of Chimonanthus salicifolius, for mouse axillary SLN mapping.

Methods and results: To improve the water solubility and SLN targeting of the chlorophyll, we encapsulated the chlorophyll in nanoscale liposomes. The liposome-coated chlorophyll nanocomposites obtained were spherical in shape and had an average diameter of $21.7 \pm 6.0 \mathrm{~nm}$. The nanocomposites dispersed well in water, and in aqueous suspension they exhibited brighter near infrared fluorescence than chlorophyll alone. After incubation of the nanocomposites with normal liver cells (QSG-7701) and macrophage cells (Ana-1) for no more than 48 hours, there was no obvious reduction in cell viability. When the nanocomposites were injected intradermally into the paw of a mouse, the axillary SLN was found to be strongly fluorescent and was easily visualized in real time without a requirement for surgery. The intensity of the near infrared fluorescence emitted by the SLN was obviously brighter than that emitted by the SLN of another mouse that had been intradermally injected with chlorophyll alone.

Conclusion: Our data show that the liposome-coated chlorophyll nanocomposites could have great potential for clinical SLN mapping due to their lack of toxicity, bright near infrared fluorescence, and small diameter.

Keywords: chlorophyll, liposomes, nanocomposites, near infrared fluorescence, sentinel lymph node mapping

\section{Introduction}

In most cancer metastases, cancer cells migrate from the primary tumor to other parts of the body through the near regional lymph nodes. The first lymph node or group of lymph nodes in the direct lymphatic drainage pathway that extends from the site of the tumor is called the sentinel lymph node (SLN), and is used to represent the status of lymphatic spread. ${ }^{1-3}$ Decisions as to the extent of surgical treatment are usually based on whether or not patients have SLN involvement. Therefore, SLN mapping and biopsy are important techniques in cancer therapy. Reactive blue dye staining, radiocolloid tracers, and a combination of both, are techniques that are widely used for SLN mapping in the clinic. However, blue-dyed SLNs located in deep tissue can only be observed after they have been exposed to air, and more extensive surgery is therefore required to find them. The disadvantages of radiocolloid tracers include exposure of the patient to radioactivity and painful peritumoral injections of radiocolloid. ${ }^{4,5}$ 
In comparison with the above three commonly used techniques, noninvasive in vivo near infrared fluorescence imaging when used for SLN mapping has several advantages. For example, SLNs in deep tissue can be observed directly due to near infrared fluorescence without the need for excessive excision of skin and muscle, which enables rapid detection of SLNs by surgeons. In addition, the surgeon can conveniently confirm through visual inspection that all of the SLNs have been removed from the node field. ${ }^{6}$ Near infrared fluorescent reagents for SLN mapping include organic dyes (eg, indocyanine green ${ }^{7-9}$ and methylene blue $)^{10,11}$ and inorganic nanoparticles (eg, quantum dots ${ }^{12-15}$ and carbon $\operatorname{dots}^{16}$ ). Organic dyes may be favored over inorganic nanoparticles because the former have lower toxicity and quicker in vivo clearance speeds.

In the present study, we report the first use of chlorophyll extracted from the leaf of Chimonanthus salicifolius for mouse axillary SLN mapping. The choice of this Chinese herb (which is usually used to getting rid of heat in summertime and stimulating appetite) is based on its nontoxic properties. In addition, C. salicifolius has a wide distribution in the south of China. C. salicifolius is a shrub that grows up to $4 \mathrm{~m}$ tall. It has been found that chlorophyll $\mathrm{a}$ and $\mathrm{b}$ are the most common types of chlorophyll in almost all of the higher plants. ${ }^{17}$ Chlorophyll is structurally similar to porphyrin (a fluorescent dye) and has near infrared fluorescence. Additionally, chlorophyll has been registered as a food additive, and a variety of foods and beverages are permitted to contain cholorophyll. ${ }^{18}$ Therefore, chlorophyll is a safe fluorescent material which has great potential for in vivo bioimaging.

However, chlorophyll is a poorly water-soluble dye. To improve its water solubility and enable it to target SLNs for imaging, chlorophyll in this study was encapsulated into nanoscale liposomes. Liposomes are spherical vesicles made up of a lipid bilayer, and are often used as a drug delivery system. It has been reported that liposomes can serve as carriers for the delivery of diagnostic and therapeutic drugs (or agents) targeted to the lymphatic system. ${ }^{19-22}$ In our study, we used low-cost soybean lecithin as the lipid membrane when preparing the liposome-coated chlorophyll nanocomposites for SLN mapping.

\section{Materials and methods Materials}

The dry leaves of $C$. salicifolius were obtained from Tongji University-Lishui Institute (Lishui, Zhejiang Province, China). Soybean lecithin (purity $>90 \%$ ) and cholesterol were acquired from Sinopharm Chemical Reagent Co, Ltd (Shanghai, China). Nude mice aged 5-6 weeks and weighing 18-22 $\mathrm{g}$ were purchased from the Shanghai Sipper-BK Lab Animal Co Ltd (Shanghai, China). The mice were used in accordance with approved institutional protocols established by the Shanghai Department of Experimental Animal Management. Normal human liver cells (QSG-7701) and mouse macrophage cells (Ana-1) were ordered from the Chinese Academy of Sciences (Shanghai, China). RPMI-1640 culture medium and fetal calf serum were obtained from Gibco (Carlsbad, CA). 3-(4,5)-dimethylthiahiazo(-z-y1)-3,5-diphenytetrazoliumromide (MTT) was bought from Shanghai Haoran Biological Technology Co, Ltd (Shanghai, China).

\section{Extraction of chlorophyll and measurement of concentration}

We mixed $10 \mathrm{~g}$ of dry leaves of C. salicifolius with $100 \mathrm{~mL}$ of ethanol and this was subsequently shaken in a sealed conical flask for 10 hours. The mixture was filtered under vacuum and the percolating solution was centrifuged to remove impurities. The green pellucid solution obtained was then condensed using a rotary evaporator.

According to the molecular structure of chlorophyll, one chlorophyll molecule contains one magnesium atom. Therefore, the chlorophyll concentration can be accurately detected by means of its magnesium content using inductively-coupled plasma atomic emission spectrometry, and calculated using the following equation:

$$
C=\left(M_{\text {chlorophyll }} / M_{\mathrm{mg}}\right) \times c
$$

where $C$ and $c$ are the concentrations of chlorophyll and magnesium, respectively, $M_{\text {chlorophyll }}$ is the molecular weight of chlorophyll a, and $M_{\mathrm{mg}}$ is the molecular weight of magnesium.

\section{Preparation of liposome-coated chlorophyll nanocomposites}

Soybean lecithin $90 \mathrm{mg}$, cholesterol $45 \mathrm{mg}$, and $2.4 \mathrm{~mL}$ of chlorophyll dissolved in ethanol (approximately $2.0 \mathrm{mg} / \mathrm{mL}$ ) were dissolved in a round-bottomed flask containing $1 \mathrm{~mL}$ of chloroform, and dried using a rotary evaporator (RE52CS, Shanghai Yarong Chemical Equipment Co, Ltd, Shanghai, China) to remove the last traces of chloroform with nitrogen flow. Next, $2 \mathrm{~mL}$ of distilled water was added to the flask to hydrate the dry lipid film, and the mixture was gently shaken for about 10 minutes, followed by sonication for at least 90 minutes. The suspension was stored at room temperature 
for 20 hours. The precipitate of free chlorophyll in the flask bottom could be observed during the 20 hours of storage period due to its poor solubility. The upper suspension (about $1.5 \mathrm{~mL}$ ) was collected and the liposome-coated chlorophyll was thus obtained.

\section{Characterization of chlorophyll and liposome-coated chlorophyll Optical properties}

Ultraviolet-visible absorption spectra were obtained using a diode array spectrophotometer (UV-2102PC, Unico, Beijing, China) with a deuterium lamp source. Fluorescence spectra excited by $400 \mathrm{~nm}$ were measured using a fluorescence spectrometer (F-2500, Hitachi, Japan) equipped with a xenon lamp source. For the detection of fluorescence stability, the samples were placed in $1 \mathrm{~cm}$ quartz cuvettes and continuously excited at $365 \mathrm{~nm}$ over a period of one hour using an ultraviolet detector (ZF, Kanghua, Shanghai, China). During the irradiation process, the fluorescence spectra of the samples were measured. The bright fields of the chlorophyll dissolved in ethanol, chlorophyll dispersed in water, and liposome-coated chlorophyll were taken using a digital color camera (Coolpix 4300, Nikon, Japan). The fluorescent images of the aqueous samples were obtained using an in vivo imaging system (Maestro $^{\text {TM }}$, CRI Inc, Woburn, MA). The excitation and emission band pass filters were 605 and $645 \mathrm{~nm}$ (long-pass), respectively.

\section{Morphology and size distribution}

The liposome-coated chlorophyll aqueous suspensions were air-dried onto carbon-coated grids, and then examined at $80 \mathrm{kV}$ using a transmission electron microscope (TEM, JSM-6360 LV, JEOL, Tokyo, Japan). The size distributions were measured by means of TEM analysis of 122 particles. To measure the hydrodynamic size of the liposome-coated chlorophyll nanocomposites in serum, the nanocomposite precipitate was dispersed in $100 \%$ fetal calf serum, and the size was ascertained using photon correlation spectroscopy (3000HS, Malvern Instruments, Worcestershire, UK).

\section{MTT assay}

Normal liver cells (QSG-7701) were cultured on a 96-well plate using RPMI-1640 as the culture medium. The medium contained $10 \%$ fetal calf serum and $1 \%$ antibiotic-antimycotic at $37^{\circ} \mathrm{C}$ and culture plates were maintained in an incubation chamber containing $5 \% \mathrm{CO}_{2}$. Each well contained $100 \mu \mathrm{L}$ of cells. For the MTT assay, $10 \mu \mathrm{L}$ of chlorophyll and liposomecoated chlorophyll aqueous suspensions containing $0,5.8$,
11.6, 23.1, and $46.2 \mu \mathrm{g} / \mathrm{mL}$ of chlorophyll were added to the cells. After a 2-hour incubation, $10 \mu \mathrm{L}$ of MTT ( $5 \mathrm{mg}$ / $\mathrm{mL}$ ) was added to the cells. After an interval of 4 hours, the suspensions were replaced with $100 \mu \mathrm{L}$ of dimethyl sulfoxide and the cell viabilities were determined by measuring their absorbance at $490 \mathrm{~nm}$ using a Flexstation III enzyme-labeled instrument (Molecular Devices, Sunnyvale, CA). To detect viability of the macrophage cells (Ana-1), these cells were incubated with $10 \mu \mathrm{L}$ of chlorophyll and liposome-coated chlorophyll (containing 0, 5.8, 11.6, 23.1, and 46.2 $\mu \mathrm{g} / \mathrm{mL}$ of chlorophyll) for 24,48 , and 72 hours, respectively, and their viabilities were measured using the same methods as described above.

\section{Animal experiments}

Chlorophyll $40 \mu \mathrm{L}$ and liposome-coated chlorophyll aqueous suspensions (containing $1.5 \mathrm{mg} / \mathrm{mL}$ of chlorophyll) were injected into two nude mice through the tail vein. Mouse fluorescence images were obtained using the in vivo imaging system (NightOWL LB983, Berthold Technologies, Bad Wildbad, Germany). The excitation wavelength was $630 \mathrm{~nm}$ and the emission filter was $700 \mathrm{~nm}$ (long-pass), and the exposure time was 0.1 seconds.

We then used the fluorescence of chlorophyll for mouse SLN mapping. The experimental details were as follows. Chlorophyll $80 \mu \mathrm{L}$ and liposome-coated chlorophyll aqueous suspensions (containing $1.48 \mathrm{mg} / \mathrm{mL}$ of chlorophyll) were injected intradermally into the paws of two nude mice. All of the injection methods (including injection depth and angle related to the plane of the paw) were the same. Fluorescence images were obtained using the Maestro ${ }^{\mathrm{TM}}$ in vivo imaging system described earlier. The excitation wavelength was $635 \mathrm{~nm}$ and the emission wavelength was $675 \mathrm{~nm}$ (long-pass). The exposure time was $500 \mathrm{msec}$. All of the collected images were analyzed using the Maestro software provided with the system. After the in vivo imaging had been completed, the lymph nodes in the axillary location at the injection sites were resected for fluorescence imaging. As a control, a lymph node in the axillary location of a mouse that had not been injected with chlorophyll was also resected for fluorescence imaging.

\section{Results and discussion}

The final extract dissolved in ethanol was green in color (Figure 1A), which indicates that it absorbed blue and red light. Its absorption spectrum exhibited two main absorption bands, ie, $<500 \mathrm{~nm}$ (blue) and 600-700 $\mathrm{nm}$ wavelengths (Figure 1B), and three peaks located at 666, 615, and $415 \mathrm{~nm}$ 
A

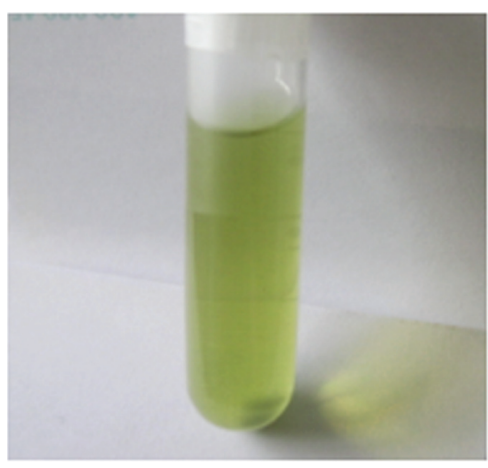

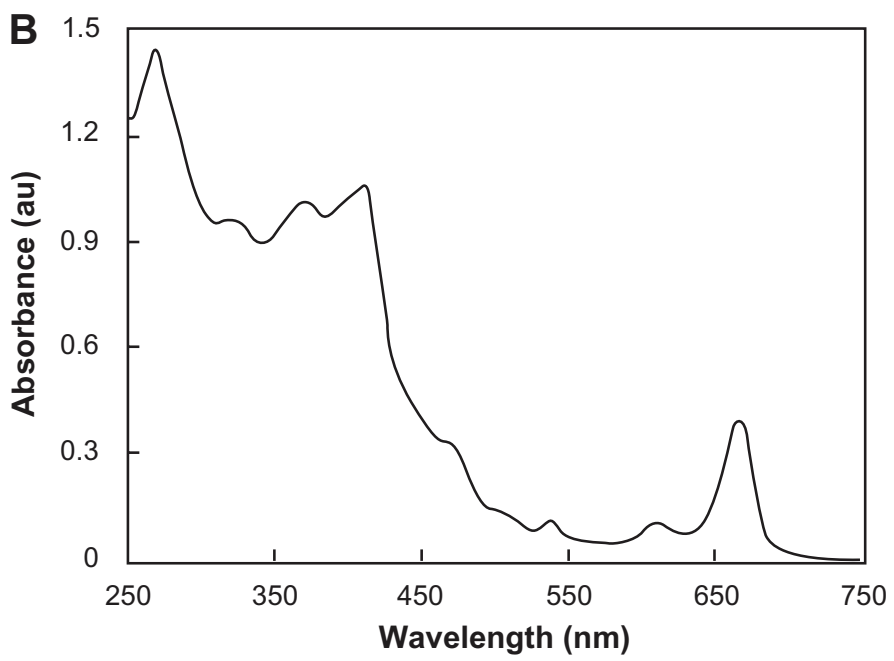

Figure I Chlorophyll dissolved in ethanol. (A) Bright field image taken using a digital color camera and (B) absorption spectrum.
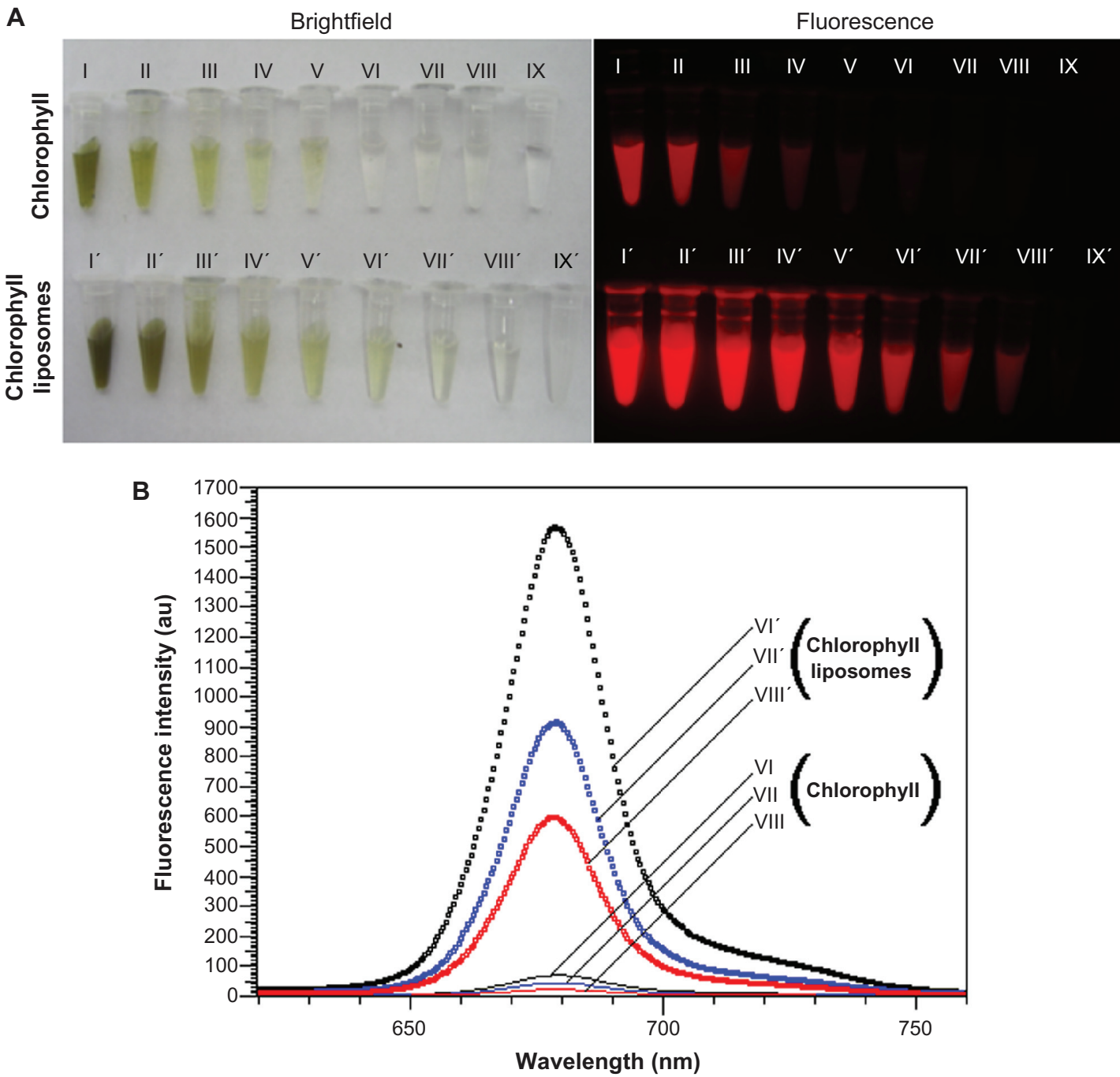

Figure 2 Bright field and fluorescence of chlorophyll dispersed in water before and after liposome encapsulation. (A) Bright field images taken using a digital color camera and fluorescent images taken using the in vivo imaging system: exciting filter $605 \mathrm{~nm}$; emission filter $645 \mathrm{~nm}$ (long-pass), and (B) fluorescent spectra excited by $400 \mathrm{~nm}$. Notes: Both chlorophyll and liposome-coated chlorophyll nanocomposites are dispersed in water. The concentrations of chlorophyll are as follows: (I and I') I.48 $\mathrm{mg} / \mathrm{mL}$, (II and II') $739.2 \mu \mathrm{g} / \mathrm{mL}$, (III and III') $369.6 \mu \mathrm{g} / \mathrm{mL}$, (IV and IV') $184.8 \mu \mathrm{g} / \mathrm{mL}$, (V and V') $92.4 \mu \mathrm{g} / \mathrm{mL}$, (VI and VI') $46.2 \mu \mathrm{g} / \mathrm{mL}$, (VII and VII) $23 . \mathrm{I} \mu \mathrm{g} / \mathrm{mL}$, (VIII and VIII) II.6 $\mu \mathrm{g} / \mathrm{mL}$, (IX) distilled water, and (IX') empty liposome aqueous suspension. 


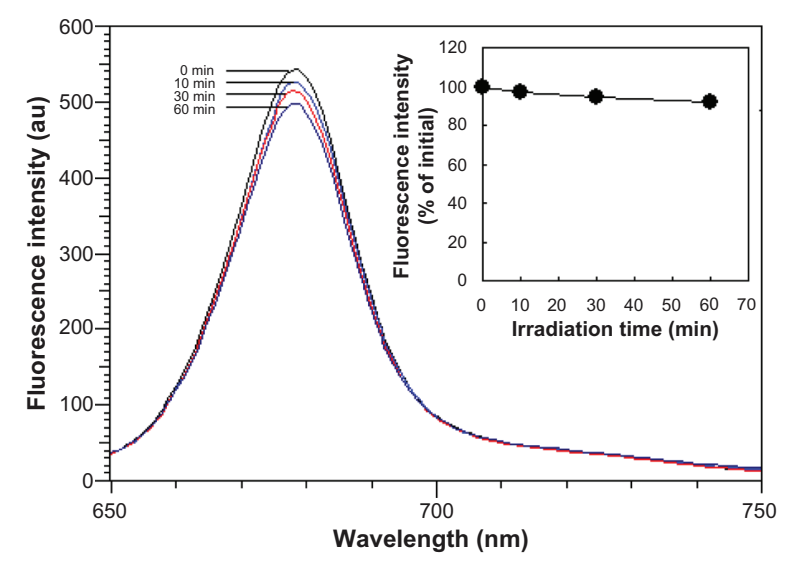

Figure 3 Photostability over time of water-dispersed liposome-coated chlorophyll nanocomposites with continuous excitation at $365 \mathrm{~nm}$ (insert, fluorescent intensity varying with excitation time).

which were well matched with the absorption spectra of chlorophyll a. ${ }^{17,23}$ C. salicifolius is a taller plant, and such plants have abundant chlorophyll a and b. ${ }^{17}$

The bright field colors of the liposome-coated chlorophyll aqueous suspensions were deeper than those of the chlorophyll aqueous suspensions alone (Figure 2A). Inductively-coupled plasma atomic emission spectrometric analysis showed that $1 \mathrm{mg}$ of liposomes could load $21.9 \mu \mathrm{g}$ of chlorophyll. An interesting finding was that the fluorescent intensities of liposome-coated chlorophyll were obviously higher than those of chlorophyll alone (Figure 2B). For example, when the concentrations of chlorophyll were $46.2,23.1$, and $11.6 \mu \mathrm{g} / \mathrm{mL}$, the fluorescent intensities of liposome-coated chlorophyll nanocomposites were 22.2, 20.8, and 25.0 times higher, respectively, than those of the chlorophyll alone (Figure 2B). The main reason for this may be as follows. Chlorophyll is a lipophilic material and has low solubility in water. However, chlorophyll molecules can be highly dispersed within the long chains of fatty acids in the lipid membrane of the liposome. Therefore, when the chlorophyll was dispersed in water, we found that the aqueous suspensions of chlorophyll contained visible particles that would precipitate in several hours. On the other hand, the aqueous suspensions of the liposome-coated chlorophyll nanocomposite were uniform, and no obvious precipitates could be observed over a week. Chlorophyll when
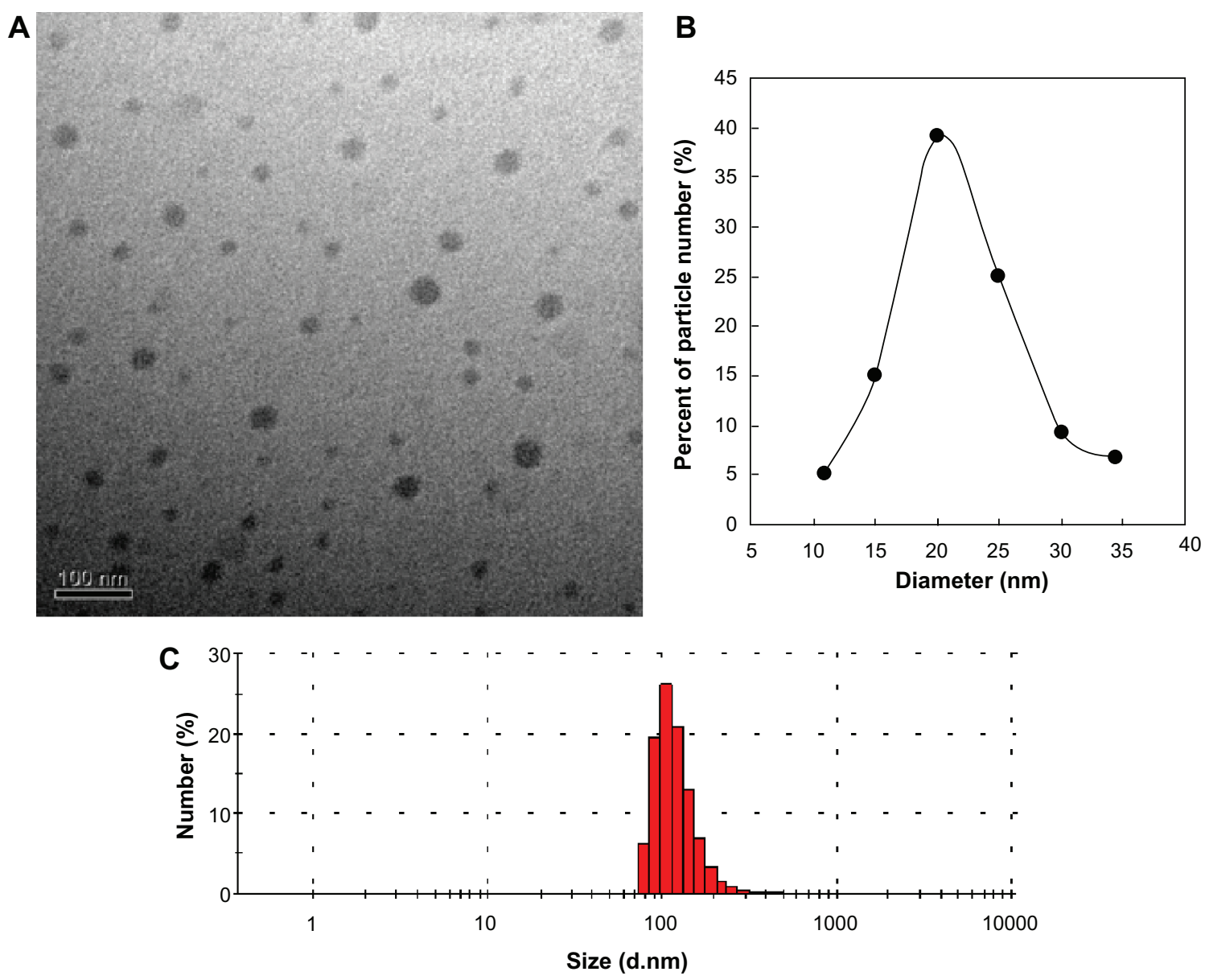

Figure 4 (A) Transmission electron microscopic image and (B and C) size distribution of liposome-coated chlorophyll nanocomposites. (B) Size distribution measured by transmission electron microscopy. (C) Hydrodynamic size distribution in serum. 
QSG-7701 cells, 2 h

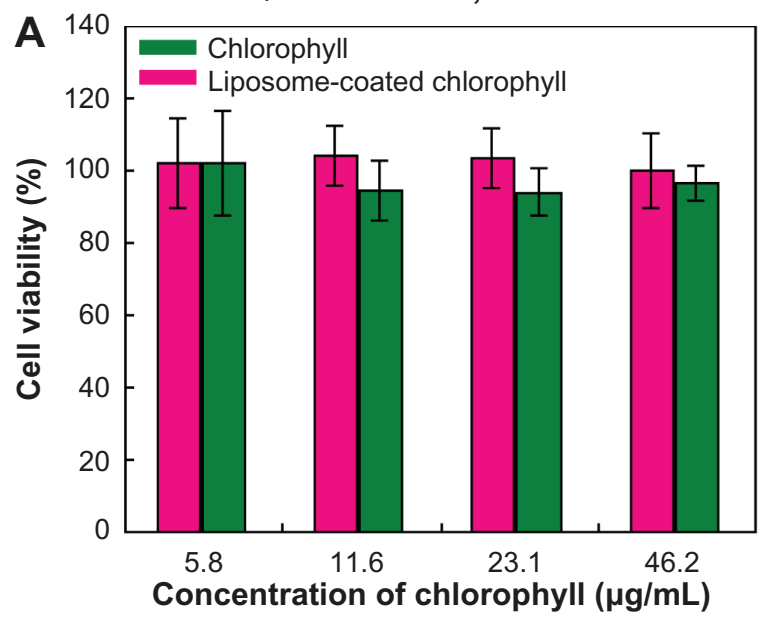

Ana-1 cells, $48 \mathrm{~h}$

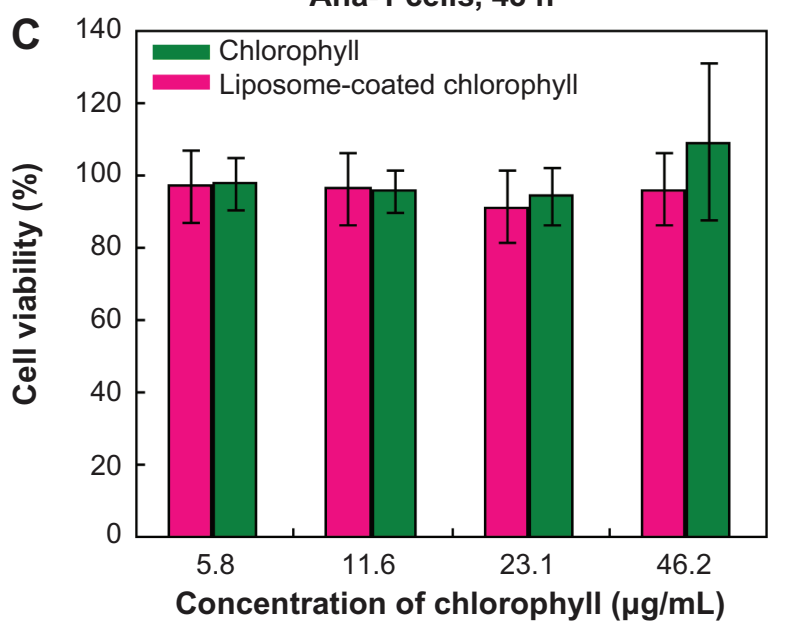

Ana-1 cells, 24 h

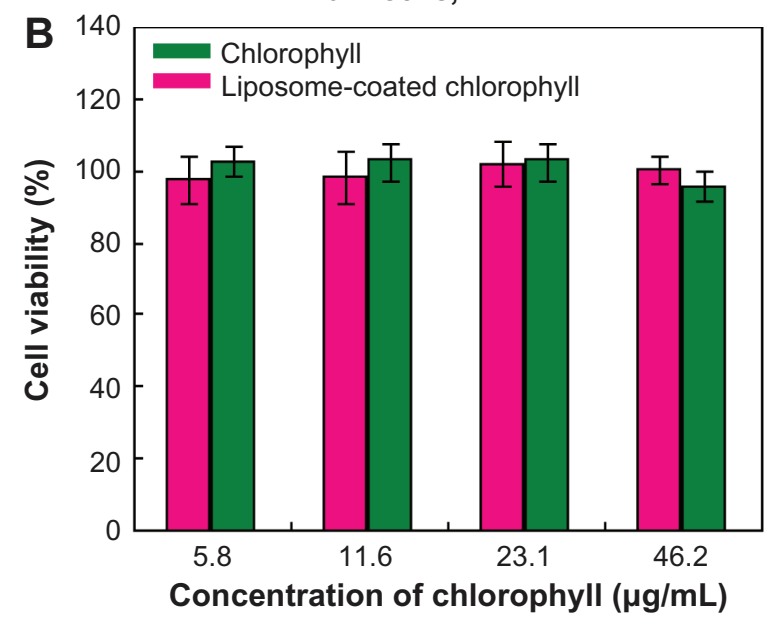

Ana-1 cells, $72 \mathrm{~h}$

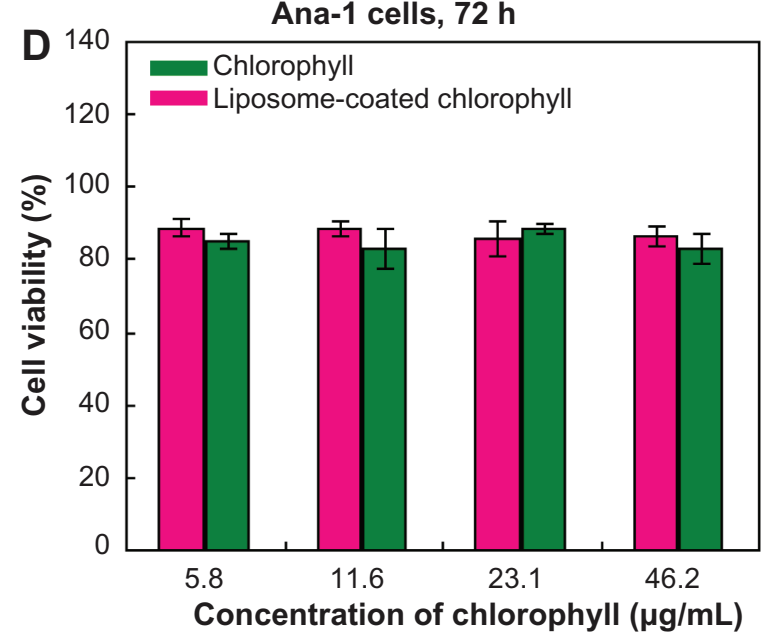

Figure 5 Viability of liver cells (QSG-770I) and macrophage (Ana-I) cells after incubation with chlorophyll and liposome-coated chlorophyll nanocomposites for different time periods.

dispersed in water has near infrared fluorescence at a wavelength of $679 \mathrm{~nm}$. After liposome encapsulation, the fluorescent peaks of liposome-coated chlorophyll nanocomposites shifted to blue by only 1-2 nm, as compared with those of chlorophyll alone (Figure 2B). This suggests that the liposome coating did not obviously affect the near infrared fluorescence of chlorophyll when penetrating deep animal tissue.

The photostability of dyes is an important factor in biomedical optical imaging. Since the chlorophyll tends to precipitate in water, here we only measured the fluorescent stability of the liposome-coated chlorophyll nanocomposites in water. After being continuously excited by a $365 \mathrm{~nm}$ light for 60 minutes, the fluorescent spectrum of the nanocomposites did not shift, and the fluorescent intensity decreased by only $8.2 \%$ (Figure 3). This indicated that the liposome-coated chlorophyll nanocomposites have potential for comparatively long-term imaging, which is of benefit for biomedical applications.
The TEM image shows that the liposome-coated chlorophyll nanocomposites are spherical in shape and have a comparatively narrow size distribution (average diameter $21.7 \pm 6.0 \mathrm{~nm}$, Figure 4A and B). Nanocomposites with such a small diameter are suitable for SLN mapping because the ideal contrast agent should be $10-50 \mathrm{~nm}$ in size. ${ }^{24,25}$ The chlorophyll molecules may be well dispersed in the liposome vesicles because chlorophyll exists in water as crystals, and virtually no free crystals could be observed around the liposome-coated chlorophyll nanocomposites. Although the average hydrodynamic size of the liposome-coated chlorophyll nanocomposites in $100 \%$ fetal calf serum is $263.4 \pm 24.0 \mathrm{~nm}$ (Figure 4C), liposomes are limp nanoparticles and may penetrate small pores in vivo. For example, to prepare different-sized liposomes, large liposomes are usually extruded in turn through polycarbonate membrane filters with different pore diameters (eg, 450, 220, and $150 \mathrm{~nm}$ ). 

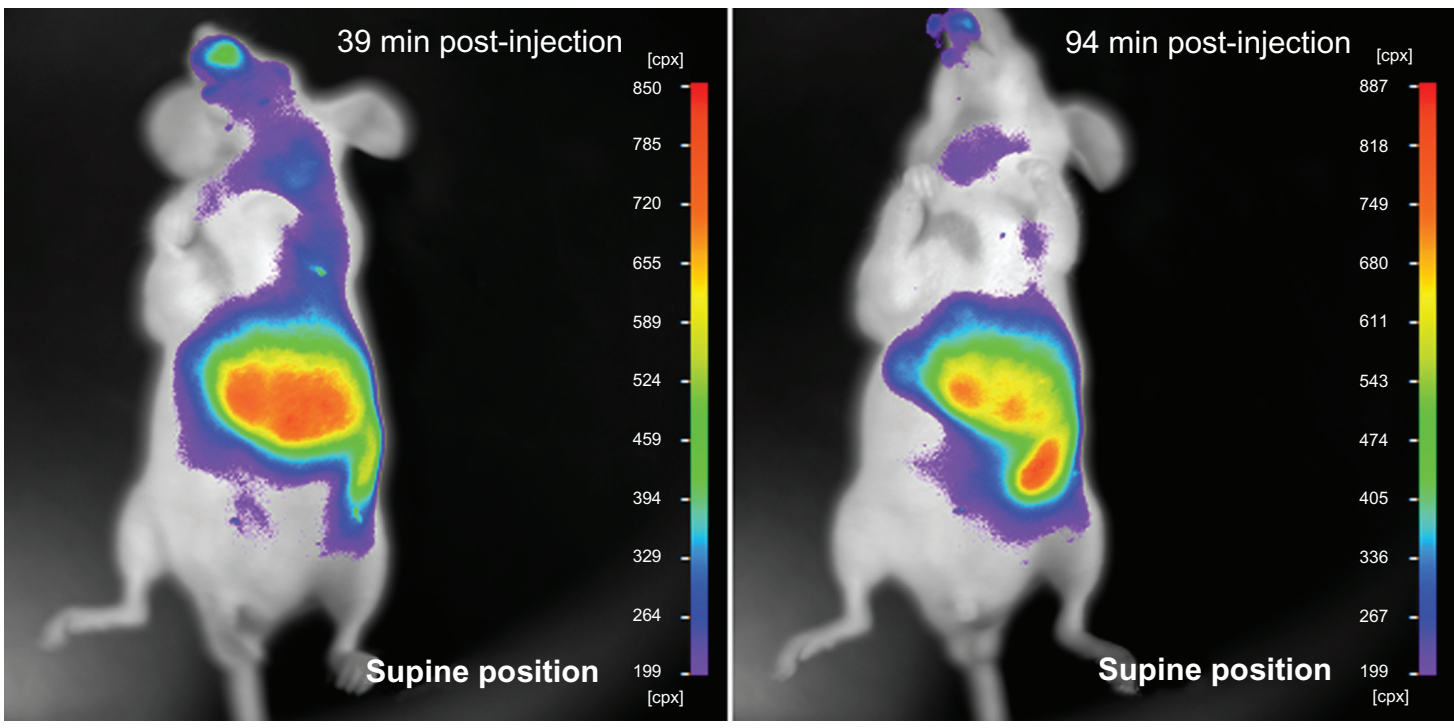

Figure 6 Liposome-coated chlorophyll nanocomposites eliminated by the animal body.

Note: The nude mouse has been injected with liposome-coated chlorophyll nanocomposites via tail vein, and its fluorescence was imaged using an in vivo imaging system.

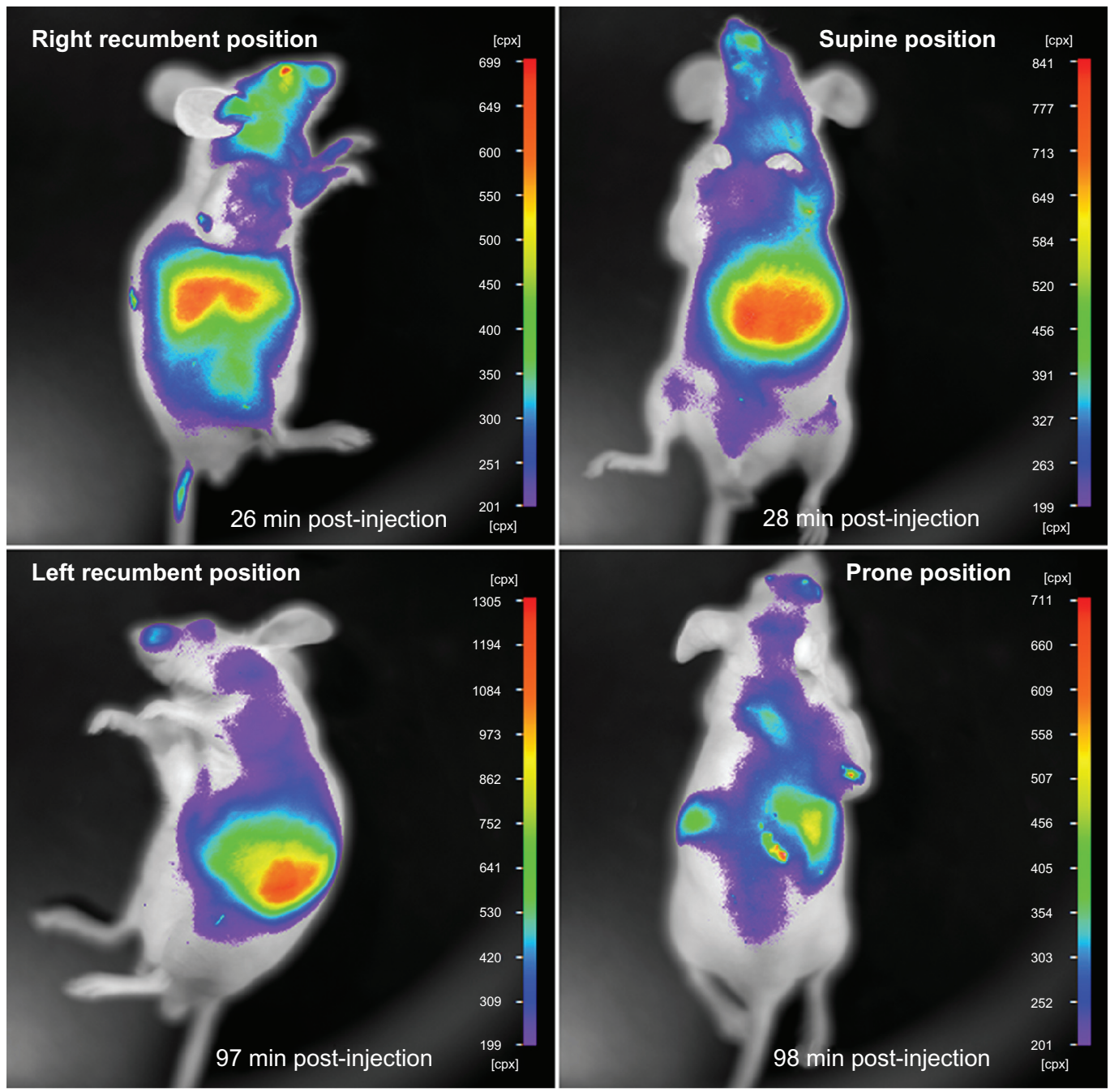

Figure 7 In vivo imaging of a nude mouse at different positions

Note: The nude mouse has been injected with liposome-coated chlorophyll nanocomposites via the tail vein. 
The advantage of chlorophyll for biomedical imaging is its low toxicity in cells. As shown in Figure 5, the MTT assay demonstrated that normal liver cell (QSG-7701) viability did not decrease obviously after the cells had been incubated with either chlorophyll or liposome-coated chlorophyll nanocomposites for 2 hours. The viability of the macrophages (Ana-1) was also not obviously affected by the chlorophyll and liposome-coated chlorophyll nanocomposites after the Ana- 1 cells had been incubated with these materials for 24 and 48 hours. It should be noted that Ana-1 cell viability was between $83.1 \% \pm 4.4 \%$ and $88.7 \% \pm 2.3 \%$ when the cells were incubated with chlorophyll and liposome-coated chlorophyll nanocomposites, respectively, for 72 hours. This indicates that the chlorophyll may exhibit low toxicity if this material exists in cells for
3 days. However, liposome nanoparticles can be eliminated from the animal body. In this work, we found that many liposome-coated chlorophyll nanocomposites might be removed from the mouse body 94 minutes after intravenous injection via the tail. This elimination process can be observed clearly through the fluorescence of liposome-coated chlorophyll nanocomposites in vivo (Figure 6). This suggests that the liposome-coated chlorophyll nanocomposites may be safely used in clinical imaging because they can be metabolized by the animal body.

In addition, the near infrared fluorescence of liposomecoated chlorophyll nanocomposites inside the mouse body could be seen at different sites through the skin and muscle after the mouse had been injected with these nanocomposites via the tail vein (Figure 7). For example, the fluorescence of

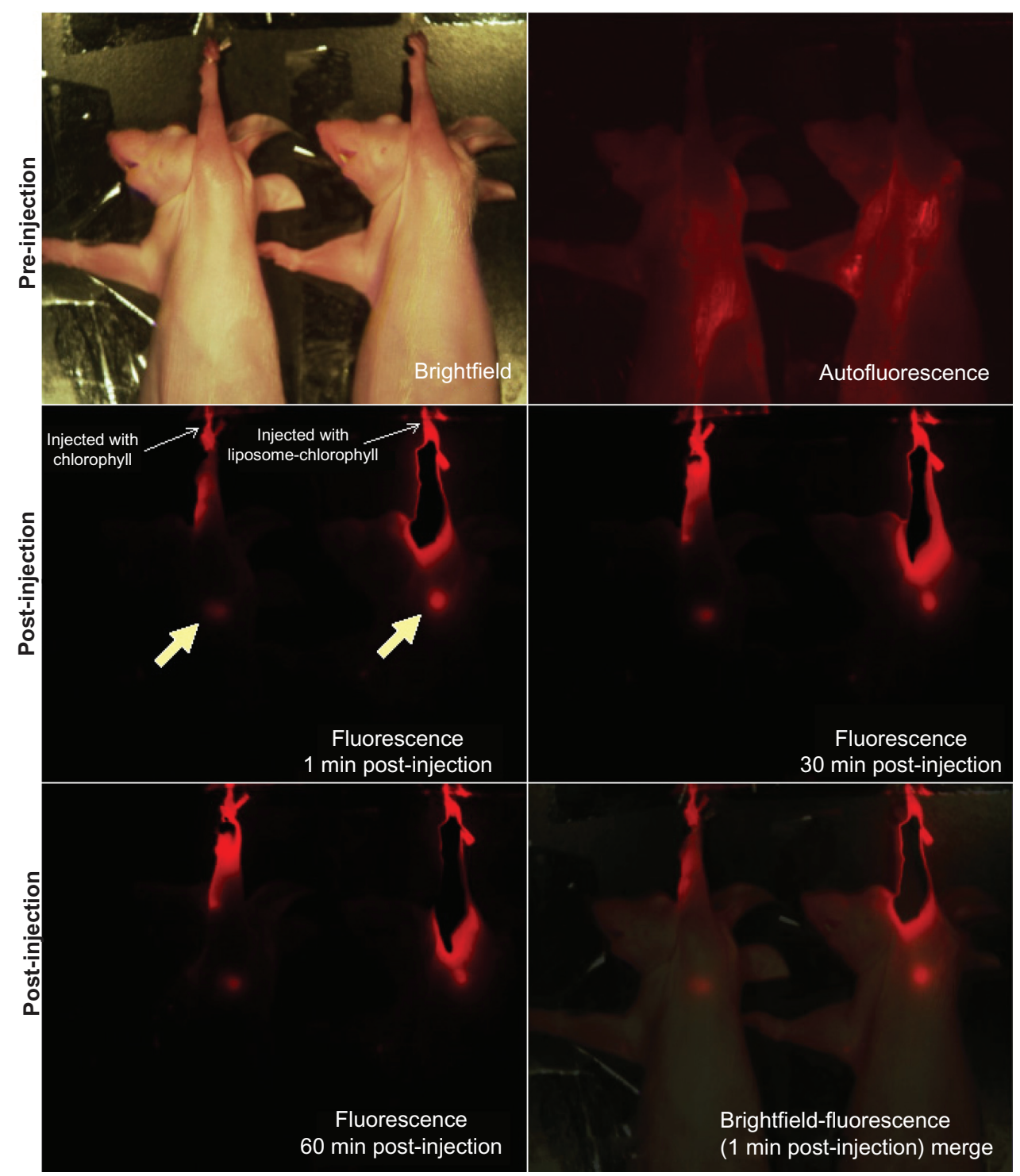

Figure 8 Sentinel lymph node mapping by means of near infrared fluorescence of chlorophyll and liposome-coated chlorophyll nanocomposites. 


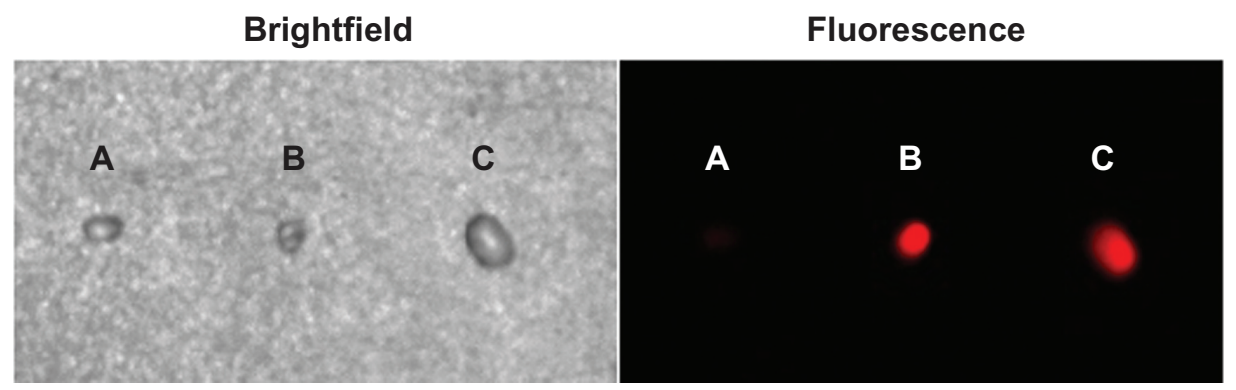

Figure 9 Bright-field and fluorescent images of the sentinel lymph nodes resected from mice that were not injected with liposome-coated chlorophyll or chlorophyll alone (A), injected with liposome-coated chlorophyll (B), and chlorophyll alone (C).

these nanocomposites in the mouse liver could be captured by the in vivo imaging system from the right recumbent, left recumbent, supine, and prone positions. Therefore, the depth of tissue penetration of chlorophyll near infrared fluorescence may be at least $3-5 \mathrm{~mm}$.

Axillary and cervical lymph nodes are usually located at deeper subcutaneous positions, so that near infrared fluorescence imaging is suitable for mapping of these lymph nodes. In this work, we injected $80 \mu \mathrm{L}$ of liposome-coated chlorophyll aqueous suspension containing $1.5 \mathrm{mg} / \mathrm{mL}$ of chlorophyll intradermally into a nude mouse paw. We then monitored migration of the chlorophyll into the axillary SLN by means of near infrared fluorescence using an in vivo imaging system. As a control, the paw of another nude mouse was synchronously injected with $80 \mu \mathrm{L}$ of liposome-free chlorophyll aqueous suspension, also containing $1.48 \mathrm{mg} / \mathrm{mL}$ of chlorophyll, and then monitored using the same methods as described above. As shown in Figure 8, the axillary region emitted bright fluorescence one minute after injection of liposome-coated chlorophyll nanocomposites. This fluorescent spot could still be observed clearly in real time at 60 minutes after injection, without the need to excise skin and muscle. The axillary region of another mouse also emitted fluorescence a short time after being injected with the liposome-free chlorophyll, but the intensity of the fluorescence was lower than that in the axillary region of the mouse injected with the liposome-coated chlorophyll nanocomposite. To verify whether the fluorescent spots were SLNs, we resected the SLNs at approximately 2 hours after injection and then imaged them using the in vivo imaging system (Figure 9). The excised SLNs emitted a bright red fluorescence. The SLN from the mouse injected with liposome-coated chlorophyll was brighter than that from the mouse injected with chlorophyll alone. This may have been because the liposome-coated chlorophyll nanocomposites had brighter fluorescence than the chlorophyll alone, and more chlorophyll was targeted at the axillary lymphatic system due to liposome delivery. This phenomenon may be very important for the surgeon because the SLNs can be observed clearly in real time. The bright red fluorescence emitted by the SLNs must originate from the chlorophyll, because the SLN of a control mouse that was not injected with chlorophyll did not emit red fluorescence.

\section{Conclusion}

In summary, chlorophyll extracted from the leaves of C. salicifolius was encapsulated into liposomes and used for the first time in SLN mapping. Due to the fact that the lipophilic chlorophyll can be well dispersed in liposomes, the liposome-coated chlorophyll nanocomposites not only improved the solubility of the poorly water-soluble chlorophyll, but also significantly improved the near infrared fluorescence of the chlorophyll. No or low toxicity was detected after incubation of cells with chlorophyll for 3 days, whether alone or encapsulated in liposomes. Although the mouse axillary SLNs can be observed to fluoresce rapidly after injection of either chlorophyll or liposome-coated chlorophyll nanocomposites, the SLN of the mouse injected with liposome-coated chlorophyll emitted brighter fluorescence than that of the mouse injected with chlorophyll alone. The SLNs embedded in deep tissues could be observed directly through near infrared fluorescence without the need for exposure of the SLNs to air. On the basis of these findings, it is believed that the use of near infrared fluorescence from liposome-coated chlorophyll nanocomposites has clinical promise for SLN mapping.

\section{Acknowledgments}

We thank Professor Xiaodong Cheng for providing the leaves of C. salicifolius. This work was supported in part by the National Natural Science Foundation of China (grant 30870711).

\section{Disclosure}

The authors report no conflicts of interest in this work. 


\section{References}

1. Morton DL, Wen DR, Wong JH, Economou JS, Cagle LA, Storm FK. Technical details of intraoperative lymphatic mapping for early stage melanoma. Arch Surg. 1992;127(4):392-399.

2. Levenback C, Coleman RL, Burke TW, et al. Intraoperative lymphatic mapping and sentinel node identification with blue dye in patients with vulvar cancer. Gynecol Oncol. 2001;83(2):276-281.

3. Levenback C. Update on sentinel lymph node biopsy in gynecologic cancers. Gynecol Oncol. 2008;111(Suppl 2):S42-S43.

4. Somasundaram SK, Chicken DW, Keshtgar MRS. Detection of the sentinel lymph node in breast cancer. Br Med Bull. 2007;84(1):117-131.

5. Kelley LM, Holmes DR. Tracer agents for the detection of sentinel lymph nodes in breast cancer: current concerns and directions for the future. J Surg Oncol. 2011;104:91-96.

6. Uren RF. Cancer surgery joins the dots. Nat Biotechnol. 2004;22(1): 38-39.

7. Kitai T, Inomoto T, Miwa M, Shikayama T. Fluorescence navigation with indocyanine green for detecting sentinel lymph nodes in breast cancer. Breast Cancer. 2005;12(5):211-215.

8. Sugie T, Kassim KA, Takeuchi M, et al. A novel method for sentinel lymph node biopsy by indocyanine green fluorescence technique in breast cancer. Cancers. 2010;2(2):713-720.

9. Yuasa Y, Seike J, Yoshida T, et al. Sentinel lymph node biopsy using intraoperative indocyanine green fluorescence imaging navigated with preoperative CT lymphography for superficial esophageal cancer. Ann Surg Oncol. 2012;19(2):486-493.

10. Chu MQ, Wan YH. Sentinel lymph node mapping using near-infrared fluorescent methylene blue. J Biosci Bioeng. 2009;107(4):455-459.

11. Chu MQ, Xiao X, Ma JY, et al. In vivo real-time near-infrared fluorescent mapping of sentinel lymph nodes using methylene blue encapsulated in a microemulsion nanosystem. Curr Nanosci. 2010;6(4):388-396.

12. Kim S, Lim YT, Soltesz EG, et al. Near-infrared fluorescent type II quantum dots for sentinel lymph node mapping. Nat Biotechnol. 2004;22(1):93-97.

13. Chu MQ, Zhuo S, Xu J, Sheng Q, Hou SK, Wang RF. Liposome-coated quantum dots targeting the sentinel lymph node. J Nanopart Res. 2010;12(1):187-197.
14. Ballou B, Ernst LA, Andreko S, et al. Sentinel lymph node imaging using quantum dots in mouse tumor models. Bioconjug Chem. 2007;18(2):389-396.

15. Knapp DW, Adams LG, Degrand AM. Sentinel lymph node mapping of invasive urinary bladder cancer in animal models using invisible light. Eur Urol. 2007;52(6):1700-1708.

16. Yang ST, Cao L, Luo PG, et al. Carbon dots for optical imaging in vivo. J Am Chem Soc. 2009;131(32):11308-11309.

17. TutorVista.com. Absorption spectrum of chlorophyll. Available from: http://www.tutorvista.com/biology/absorption-spectrum-ofchlorophyll. Accessed February 25, 2012.

18. Adams J. Hideous Absinthe: A History of the Devil in a Bottle. Madison, WI: University of Wisconsin Press; 2004.

19. Zavaleta CL, Phillips WT, Soundararajan A, et al. Use of avidin/ biotin-liposome system for enhanced peritoneal drug delivery in an ovarian cancer model. Int J Pharm. 2007;337(1-2):316-328.

20. Oussoren $\mathrm{CH}$, Storm G. Liposomes to target the lymphatics by subcutaneous administration. Adv Drug Deliv Rev. 2001;50(1-2):143-156.

21. Oussoren C, Storm G. Targeting to lymph nodes by subcutaneous administration of liposomes. Int J Pharm. 1998;162(1-2):39-44.

22. Oussoren C, Zuidema J, Crommelin DJA, Storm G. Lymphatic uptake and biodistribution of liposomes after subcutaneous injection. II. Influence of liposomal size, lipid composition and lipid dose. Biochim Biophys Acta. 1997;1328(2):261-272.

23. Brody SS, Broyde SB. Low temperature absorption spectra of chlorophyll a in polar and nonpolar solvents. Biophys J. 1968;8(12): 1511-1533.

24. Josephson L, Mahmood U, Wunderbaldinger P, Tang Y, Weissleder Y. Pan and sentinel lymph node visualization using a near-infrared fluorescent probe. Mol Imaging. 2003;2(1):18-23.

25. Ohnishi S, Lomnes SJ, Laurence RG, et al. Organic alternatives to quantum dots for intraoperative near-infrared fluorescent sentinel lymph node mapping. Mol Imaging. 2005;4:172-181.
International Journal of Nanomedicine

\section{Publish your work in this journal}

The International Journal of Nanomedicine is an international, peerreviewed journal focusing on the application of nanotechnology in diagnostics, therapeutics, and drug delivery systems throughout the biomedical field. This journal is indexed on PubMed Central, MedLine, CAS, SciSearch $\AA$, Current Contents ${ } /$ Clinical Medicine,

\section{Dovepress}

Journal Citation Reports/Science Edition, EMBase, Scopus and the Elsevier Bibliographic databases. The manuscript management system is completely online and includes a very quick and fair peer-review system, which is all easy to use. Visit http://www.dovepress.com/ testimonials.php to read real quotes from published authors. 